INTERNATIONAL DESIGN CONFERENCE - DESIGN 2018

https://doi.org/10.21278/idc.2018.0243

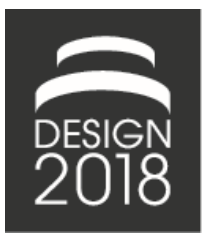

\title{
BIG DATA ANALYSIS AS A NEW APPROACH FOR USABILITY ATTRIBUTES EVALUATION OF USER INTERFACES: AN AUTOMOTIVE INDUSTRY CONTEXT
}

\author{
J. Orlovska, C. Wickman and R. Söderberg
}

\begin{abstract}
The growth of digital technologies dramatically increased the complexity of user interfaces in modern cars that made the usability evaluation an arduous task. Current usability evaluation methods cannot provide sufficient information about a user and system performance for such a complex and dynamic environment as automotive user interfaces. In this paper, we investigate how and to what extent the big data analysis can contribute to the usability attributes evaluation. We present the case study design for usability evaluation of Driver Support system, validated by the industry professionals.
\end{abstract}

Keywords: big data analysis, artificial intelligence (AI), user experience, usability, user interface

\section{Introduction}

The complexity of the real driving environment is a continuous challenge for the design of HMI (Human-Machine Interface) systems, as dynamically changing scenarios are randomly induced in the real driving environment. These scenarios generate infinite interaction variations and make evaluation processes obscure. It is even more challenging to perform a prognosis of the user's individual reactions to these scenarios. The multi-layered user interfaces' hierarchy, along with a combination of physical and graphical elements, encourages drivers to experiment with HMI, applying different scenarios, creating their own real-time driving experience (Chalmers, 2004). Along with a continually decreasing time-frame of new product development processes in the automotive industry (Diaby et al., 2013) usability evaluation is currently an extremely arduous task.

Henceforth, usability engineers today need to apply a multidisciplinary approach, including expertise from a wide range of research areas, such as engineering, computer science, psychology, sociology, and neuroscience (Law et al., 2009). This has resulted in a variety of usability assessment methods with heterogeneous backgrounds. According to ISO 9241-11, usability is defined as "the extent to which a product can be used by specified users to achieve specified goals with effectiveness, efficiency, and satisfaction in a specified context of use". Therefore, this definition plays a key role in the usability evaluation.

According to Ivory and Hearst (2001), there are 132 documented Usability Evaluation Methods (UEMs). Some of these are suitable for applying at the early stages of design, e.g., heuristic evaluation. Some of them can be applied only at the prototype phase, or even after the interface design has been implemented. Unfortunately, the majority of existing methods for usability evaluation of released products are based on inquiry methods such as surveys, questionnaires or interviews. Those methods presume the use of subjective ratings with usability ranking scales and focus on the gathering of 
subjective impressions regarding the HMI usage, rather than focusing on specific user tasks or measuring user performance.

However, the user performance metrics are a most essential part of the usability evaluation (Albert and Tullis, 2013). The use of big data in this case can help to achieve more reliable results on performance measuring, because of the ability to gather the variety of driving parameters in the real driving environment. Existing automotive platforms today generate an enormous amount of data. Nevertheless, big data is not knowledge - there is a need in the development of methodologies for classification and sophisticated extraction of the relevant information regarding usability evaluation. It is essential to add that big-data analysis does not aim to replace existing subjective methods for usability evaluation, but instead aims to augment. Currently big data analysis is able to detect only fragmentary areas of usability attributes, excluding those related to the user's perceptions induced by the use of the interface.

To investigate the applicability of real user data analysis for the usability attributes assessment, we performed a study that included interviews with the usability engineers from one of the leading Swedish automotive OEMs (Original Equipment Manufacturer). We identified the list of usability attributes involved in the evaluation of the Driver Support system. The Driver Support system is a semiautonomous system that can control the vehicle with regard to steering, braking or accelerating through the various traffic conditions, as well as provide help in controlling the vehicle on the road. Consequently, we organized focus group discussions with the purpose of compiling the identified usability attributes list and evaluate possible pitfalls of usability assessment for the released product. Furthermore, we investigated the possibility of big data analysis involvement in the assessment of usability attributes. We also disclosed evidence that subjective usability attributes assessment combined with real-time data analysis can significantly improve current usability attributes evaluation practices. Incorporating realtime data analysis into the usability attributes assessment can increase the usability issues detection and allow their magnitude to be measured. The obtained qualitative results suggest further research is warranted, and a framework is proposed to use real user data analysis for usability evaluation.

The particular framework was developed to endorse the Driver Support system evaluation. Data mining and acquisition of test vehicles in this study was supported by the WICE system (flexible automotive platform providing access to measurement data from test vehicles) and was provided by the OEM.

The remainder of the paper is structured as follows: Section 2 presents the background of relevant interdisciplinary knowledge; Section 3 introduces the methodology chapter; Section 4 discusses results of case study design, together with the qualitative findings that suggest further research; Section 5 presents the conclusions of this paper.

\section{Background}

The aim of this study is to understand how big data analysis can be used for usability attributes evaluation. It is a multidisciplinary research field, and therefore this section includes a brief description of research areas that influenced our study.

\subsection{Usability}

Usability is one of the essential factors for the success of a product. Interaction with a product that is easy to use and understand increases users' productivity, learning pace and satisfaction with the product itself. The main advantage for users is that they can perform intended tasks naturally and efficiently. Good usability of a particular product in comparison with similar products usually means that user's loyalty will likely remain in the future. Good usability also increases the quality perception of the product and usually leads to a sales increase. Therefore, the primary goal for usability engineers is to design a system that people find usable and will use (Ovaska, 1991).

Despite the fact that the term of usability is widely in use, there is no agreement on a final definition (Paige et al., 2017). Different opinions regarding the ways to measure usability, together with the different approaches, generated many similar definitions in the field (Folmer and Bosch, 2004). The most widely accepted definition in Human-Computer Interaction (HMI) is derived from ISO/IEC 924111 standards; "the extent to which a product can be used by specified users to achieve specified goals with effectiveness, efficiency, and satisfaction in a specified context of use." Initially, usability definition focussed on the outcome of interaction rather than the quality of the product with which the 
user is interacting. In the later standards (ISO/IEC 9241-210; ISO/IEC 9241-210), however, the term usability has a twofold definition; as the quality of the product and the outcome of interaction related to its quality in use (Paige et al., 2017).

According to Bruno and Al-Qaimari (2004), usability consists of four common factors that have an impact on the whole interactive system: the user, the technology, the task, and the context of use. Consequently, we adopted this definition in our study. We assume that only understanding of the user's archetype and technical limitations of the interactive systems within the specific context of system's use, including the analysis of the influence of external conditions, can lead to the successful development of the interactive system that meets user's requirements.

According to Peham et al. (2014), Usability of User Performance could be described by the two following processes:

Learning process (represented by the Learnability attribute in Table 1) - the dynamic process that could be described as a process of gaining knowledge by studying, practicing and improving specific skills. Learning cannot be developed instantly, but develops over time as experience increases. When the Learning process comes to an end (when a user has reached stable user performance that doesn't change significantly over time), the Usage process takes place.

Usage process (represented by Ease of use attribute in Table 1) presumes that the driver has learned how to use the product and the Usage process measures how easy the product is to use, once it has been learned. Figure 1 represents the improvement of user performance skills during the learning and usage processes.

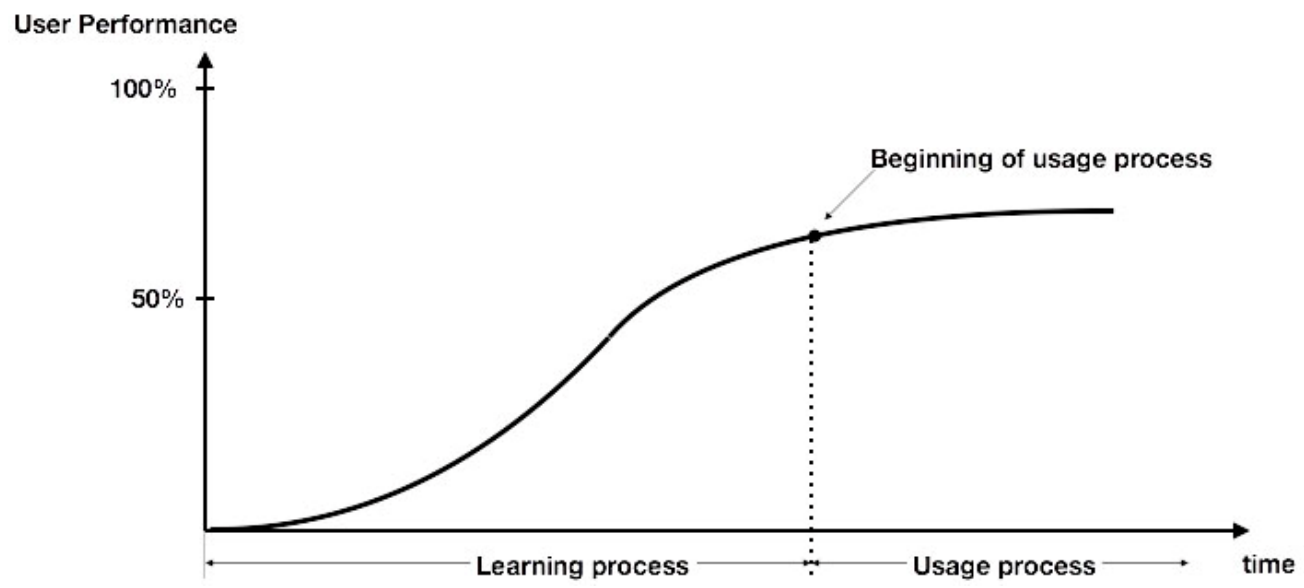

Figure 1. Learning and Usage processes in a User Performance development curve

\subsection{Usability attributes}

There have been many attempts to define the list of attributes for usability assessment. However, no agreement regarding which attributes are essential for usability evaluation has so far been reached. The digitalization trend for complex products, such as mobile phones, computers or cars, has consequently increased the convergence between computer science, telecommunication, and engineering fields. This has only boosted complexity level and introduced new types of interaction intended to help a user in communication with technology (e.g., touch screens, voice commands, gesture interfaces). Such a dramatic change in the interface frameworks forced usability engineering to engage a large number of specialists within various disciplines. As a result, the usability attributes list is usually unique for every new product development project.

According to the literature review performed by Becerril et al. (2017), sixty usability attributes were derived from numerous cases. In our case, using the OEM's internal documentation, we identified a set of sixteen attributes that are relevant for usability evaluation of the Driver Support system. Some of these are traditional usability attributes that are well described in the literature. However, a number of the attributes were derived from the OEM's internal documentation and are considered essential for usability evaluation of the Driver Support system. The list of chosen usability attributes, along with their descriptions, is compiled in Table 1. 
Table 1. List of usability attributes

\begin{tabular}{|c|c|}
\hline Style name & Style properties \\
\hline Learnability & $\begin{array}{l}\text { According to ISO/IEC 9126, Learnability is the ability to learn how to use a product or } \\
\text { system by different user groups, i.e., novice, expert, casual, etc. }\end{array}$ \\
\hline Ease of use & $\begin{array}{l}\text { The measure of how easy it is to use the product, once it has been learned. Ease of use } \\
\text { consists of following sub-attributes: Effectiveness, Efficiency, Error rate and } \\
\text { Usefulness. }\end{array}$ \\
\hline Effectiveness & $\begin{array}{l}\text { The accuracy, and completeness with which users achieve specified goals (ISO 9241- } \\
\text { 11). }\end{array}$ \\
\hline Efficiency & $\begin{array}{l}\text { Resources expended in relation to the accuracy and completeness with which users } \\
\text { achieve goals (ISO 9241-11). }\end{array}$ \\
\hline Error rate & $\begin{array}{l}\text { A frequency of errors that are made by user input or system while performing the } \\
\text { function. }\end{array}$ \\
\hline Usefulness & $\begin{array}{l}\text { The inclusion of extra components or functions of a system which are necessary for } \\
\text { functionality, in order to satisfy the user's view on interaction. In our case, we are also } \\
\text { interested in investigating the necessity and timeliness of the function itself and } \\
\text { looking at the degree to which the function is used by specific users. }\end{array}$ \\
\hline System Reliability & $\begin{array}{l}\text { The ability of a system, product or component to perform specified functions under } \\
\text { stated conditions for a specified period of time (adapted from ISO/IEC/IEEE 24765). } \\
\text { In the context of this study, "reliability is closely related to availability, which is } \\
\text { typically described as the ability of a component or system to function at a specified } \\
\text { moment or interval of time" (IEEE Std } 610.12,1990 \text { ). }\end{array}$ \\
\hline System Robustness & $\begin{array}{l}\text { The degree to which a system or component can function correctly in the presence of } \\
\text { invalid inputs or stressful environmental conditions (IEEE Std } 610.12,1990) \text {. }\end{array}$ \\
\hline $\begin{array}{l}\text { System } \\
\text { Responsiveness }\end{array}$ & $\begin{array}{l}\text { Refers to the specific ability of a system or function to complete assigned tasks within } \\
\text { a given time. }\end{array}$ \\
\hline $\begin{array}{l}\text { Holistic } \\
\text { Appearance }\end{array}$ & $\begin{array}{l}\text { The system shall be perceived as a whole regarding the graphical interface, tactile } \\
\text { feedback, sound and performance (defined by OEM). }\end{array}$ \\
\hline Consistency & $\begin{array}{l}\text { Refers to the principle that things that are related should be presented similarly and } \\
\text { things that are not related should be distinctive (Dumas and Redish, 1999). The } \\
\text { approach of consistency applies to many different aspects. Within the context of this } \\
\text { paper (final evaluation of the product) consistency presumes that the ways of } \\
\text { performing similar actions shall be perceived consistently within the system (defined } \\
\text { by OEM). }\end{array}$ \\
\hline Readability & $\begin{array}{l}\text { The ability of a user to understand a written text (Klare, 2000). Readability depends on } \\
\text { its content representation: font size, line height, etc. Within the context of this study, } \\
\text { readability is understood as a combination of text and icons that shall be easy to } \\
\text { see/read (defined by OEM). }\end{array}$ \\
\hline $\begin{array}{l}\text { Perceived driver } \\
\text { safety }\end{array}$ & $\begin{array}{l}\text { The system shall be perceived as safe to use while driving. This attribute is essential } \\
\text { for the particular system because the Driver Assist is a semi-automated system and the } \\
\text { driver shall feel safe and secure while the system is activated. }\end{array}$ \\
\hline Understandability & $\begin{array}{l}\text { The capability of the product or system to enable the user to understand how it can be } \\
\text { used for specific tasks and conditions of use (ISO/IEC 9126-1, 2001). To ensure that } \\
\text { understandability can be achieved, many aspects have to be taken into consideration: } \\
\text { user expertise, structural implementation, visual representation, and the complexity of } \\
\text { vocabulary. The evaluation elements for this study are icons, words, and acronyms that } \\
\text { shall be understandable (defined by OEM). }\end{array}$ \\
\hline Accessibility & $\begin{array}{l}\text { Refers to the degree to which information is easily accessible, having in focus people } \\
\text { who experience various types of disabilities (Henry et al., 2014). In our study, } \\
\text { evaluating the accessibility, we focus on how users perceive the level of functionality. } \\
\text { They shall perceive it naturally and sufficient for their needs (defined by OEM). }\end{array}$ \\
\hline Satisfaction & $\begin{array}{l}\text { According to Farris et al. (2010), user satisfaction can be seen as "a measure of how } \\
\text { products or services meet or surpass customer expectation". }\end{array}$ \\
\hline
\end{tabular}




\subsection{Usability evaluation methods (UEMs)}

According to Ivory and Hearst (2001), there are one hundred and thirty-two documented usability evaluation methods (UEMs), which can be divided into five classes: testing, inspiration, inquiry, analogical modeling, and simulation. Some of the methods are suitable for applying at the early stages of design, e.g., heuristic evaluation, while some are the best fit for the prototyping or user testing phase. It is important to mention that the described 132 methods originate from WIMP (windows, icons, pointer, and mouse) and Web user interfaces assessment methodologies. However, for the sophisticated interfaces assessment, including a physical interface in combination with a graphical interface placed in multi-modal screens, the number of applicable methods is insufficient.

Nielsen (1994) suggested using several evaluation methods in combination, appealing to incremental findings regarding various usability issues and cross-checking of the evaluation results. Two Comparative User Testing studies CUE-1 and CUE-2 (Molich et al., 1999) confirmed his suggestion and demonstrated the lack of consistency and systematic approach for usability evaluations. Those studies showed that usability findings performed for the same project could vary dramatically, depending on usability assessment team knowledge and the methods that usability experts chose for the evaluation. That is how the results strive towards uncertainty of outcome, which makes it difficult for the decision-making processes.

Thus, usability engineers need a methodology support to perform their assessment tasks with a higher level of objectivity. For this reason, the idea to utilize the explicit knowledge from the available variety of data is very attractive and promising for usability engineers. It could help to focus on the improvement of the user and system performance evaluation. With real data analysis it is possible to understand the user better, by looking at the learnability process dynamics, evaluating the using process, typical behavior, detecting the usability issues and measuring their magnitude.

\subsection{Big data}

Big data is a comparatively new term that indicates an amount of data that is "difficult to store, process and analyze using traditional database technologies" (Hashem et al., 2015). The definition of big data can vary from a large volume of data for scientific visualization (Cox and Ellsworth, 1997) to a large volume of data that is beyond technical capability regarding storing, managing and processing the data efficiently (Brown et al., 2011). The most traditional way to describe big data is through the "three V's" - big data characteristics: Volume, Variety, and Velocity (Berman, 2013). However, some researchers, e.g., (Gantz and Reinsel, 2011; Chen et al., 2014) argue that Value, the fourth "V"s characteristic, is the most important dimension of big data. Value extracting is the primary purpose of big data processing. It highlights the importance of big data as a source of knowledge and refers to the process of discovering hidden values from large datasets (Alpaydin, 2010).

The definition provided by Gantz and Reinsel (2011) reflects the view on big data adopted in this study: "Big data describes a new generation of technologies and architectures, designed to extract value from very large volumes of a wide variety of data, by enabling high-velocity capture, discovery, and/or analysis." Big data can include transactional data, warehoused data, metadata, and other data that could be captured from information around the product consumption or utilization (e.g., GPS data, media or infotainment data).

\subsection{Technical characteristics of the data mining system}

The case study design described in this paper includes the help of the WICE system, which allows the field evaluation of the vehicles fleet involved in the study. The WICE system is a telematics platform providing data access from the test vehicles. It consists of two major parts: (i) an in-vehicle telematics data measurement system, and (ii) a back-end server infrastructure together with the web-based frontend user interface, including data storage units and database. Overall, the system provides metrology services, including various signal types collection and measurement. The WICE system is able to manage information regarding the vehicles fleet by keeping track of map-based positioning, mileage, and uptime or diagnostic codes. Johanson (2017) gives a more detailed description of the WICE system. 


\subsection{Functional description of the Driver Support system}

The Driver Support system is a semi-autonomous system that can steer, brake or accelerate the vehicle through the various traffic conditions, as well as provide help in the control of the vehicle on the road. In the case study design, we evaluate two functions of the Driver Support system: Adaptive Cruise Control and Pilot Assist.

\subsubsection{Adaptive Cruise Control}

The ACC (Adaptive Cruise Control) function helps the driver to maintain vehicle speed with a preselected time interval to the vehicle positioned in front. ACC is usually enabled during long journeys with steady traffic conditions. This is achieved using the vehicle's cameras and radar and automatically adjusts the vehicle's speed regarding other objects moving in front.

\subsubsection{Pilot Assist}

The PA (Pilot Assist) function helps the driver to keep the car in the road lane and maintain an interval to the preceding vehicle, taking the speed control into consideration. This achieved by the usage of cameras and the vehicle's radar unit. A driver can ignore the system recommendations since it is semi-autonomous.

\section{Methodology}

The purpose of this research is to understand how real user data analysis can contribute to subjective usability attributes evaluation, and design a case study for usability assessment of the Driver Support system. To identify relevant usability attributes, we performed a study of the OEM's internal documentation. From the variety of usability attributes, a list of 16 attributes related to the Driver Support system was derived (see Table 1). The present-day assessment practices for these attributes regarding released vehicles were identified primarily as inquiry methods, e.g., surveys, questionnaires, interviews. Consequently, the first round of discussions was performed with the usability engineers to verify the obtained list of usability attributes.

At the next stage, a focus group discussion was organized and performed. We analyzed the attributes and divided them into three major groups: User Performance, System performance, and User Perception. The classification of attributes is depicted in Figure 2. Notably, the User Performance and System Performance attributes can be supported by the WICE-data. However, the User Perception attributes are not supported by the WICE-data. The reason is shortcomings regarding the ability to measure user's emotions or similar cognitive feedback by means of existing hardware. Unfortunately, real environment assessment has its limitations - it is possible to identify and analyze user activities, but not the individual affective reactions.

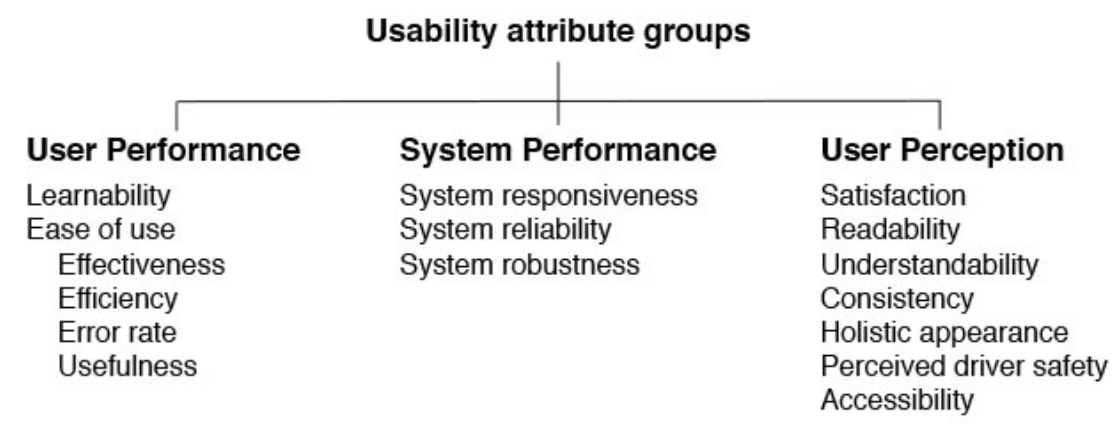

Figure 2. Usability attributes classification

In the second round of interviews, we performed unstructured face-to-face interviews with nine usability engineers involved in the user interface evaluation processes. The primary goal of the interviews was to identify measurable areas regarding the usage of the Driver Support system. As a result, we obtained a list of questions regarding usability assessment for User and System Performance groups of attributes. Furthermore, the list of questions was examined for double entries and questions were assigned to the relative usability attributes. Finally, 18 usability questions related to Driver Support system evaluation 
were listed for analysis with the help of data mining. Examples of the questions listed were "How long does it take for a user to learn what PA/ACC can and cannot do?" or "Are there any users who tested $P A$ once and never again?"

At the final stage a few workshops were organized. This time the group of participants included not only usability engineers but also data engineers and data analysts. Significant discussion was organized around the following topics:

- to identify all types of data that engineers need to answer the 18 usability questions defined in the previous steps;

- to ensure the possibility of required data retrieval (from the side of data engineers);

- to define and describe different scenarios for metrics assessment, if any;

- to define which user characteristics are essential for the case study design, if any.

Hence, the case study for data-based usability evaluation was designed.

\section{Results and discussions}

The main result of this study is a case study design for Driver Support system assessment. We propose the approach for usability attributes evaluation that is based on the integration of real user data analysis into the overall usability assessment of HMI. The design of the case study, including big data analysis for assessment of the usability attribute groups regarding the Driver Support system, is depicted in Figure 3. The study procedure was planned as follows: (i) to perform the objective evaluation, we need to set usability evaluation questions linked to the attributes list; (ii) consequently, we need to identify required data and define the data set; (iii) we need to define the measuring parameters, such as the number of trials, the time frame for measuring, specific user parameters if any; (iv) data engineers collect the required data according to measuring parameters. (v); collected data is analyzed, to be able to answer the usability questions and calculate the values of the usability attributes.

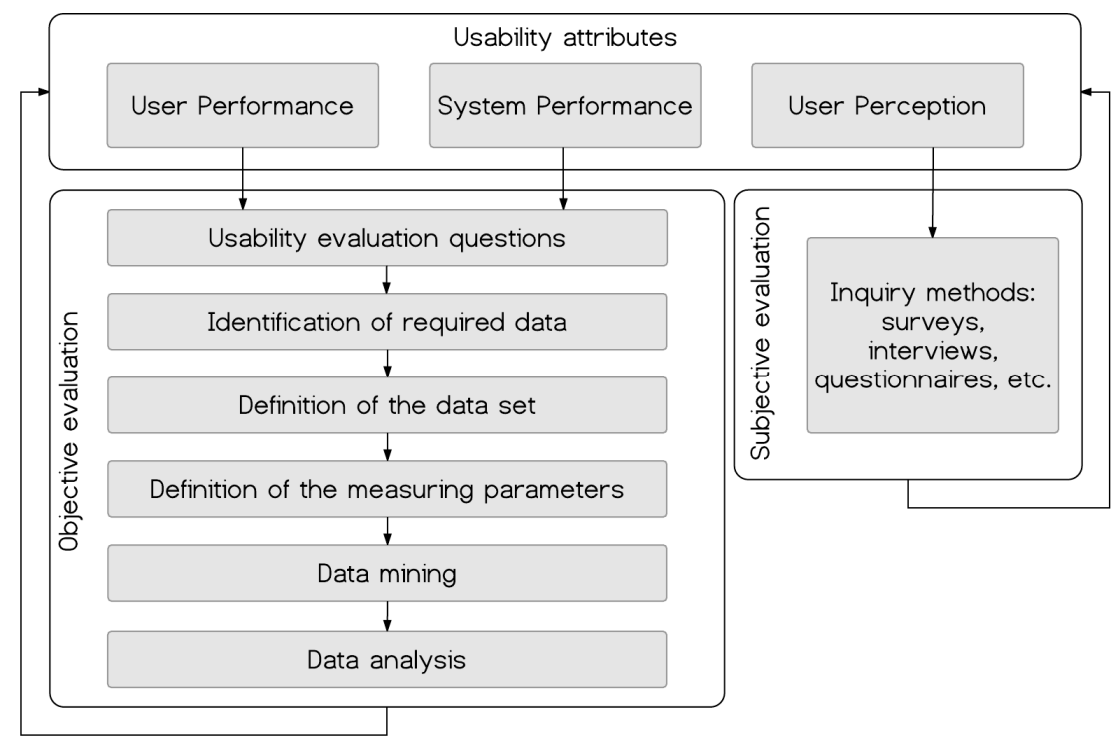

Figure 3. Case study design

Figure 4 shows an example of the "Error rate" attribute's assessment procedure with the help of the usability evaluation questions. In order to assess the "Error rate" attribute with data, we set two evaluation questions that aimed to find out what mistakes users made and how often they occurred. To answer these questions with a certain level of confidence we need to identify the vehicle ID number and to what market it belongs (e.g., Sweden, India, America) to ensure the authenticity of user data and take into consideration possible cultural issues. We also need to measure mistakes made by the same user, which is why user identification signals should be included. We also need to differentiate users' errors and count their number. 
The same approach was applied to the rest of the User and System Performance attributes that are supported by the WICE-data.

\begin{tabular}{|c|c|c|}
\hline Usability structure: & Usability questions: & Data support parameters: \\
\hline $\begin{array}{l}\text { User Performance: } \\
\text { Learnability } \\
\text { Ease of use } \\
\text { Effectiveness } \\
\text { Efficiency } \\
\text { Error rate } \\
\text { Usefulness } \\
\text { System Performance: } \\
\text { System responsiveness } \\
\text { System reliability } \\
\text { System robustness } \\
\text { User Perception: } \\
\text { Satisfaction } \\
\text { Readability } \\
\text { Understandability } \\
\text { Consistency } \\
\text { Holistic appearance } \\
\text { Perceived driver safety } \\
\text { Accessibility }\end{array}$ & $\begin{array}{l}\text { Does user appreciate PA (Pilot Assist) and use it more and more } \\
\text { the longer he/she had the car? } \\
\text { Does the number of user errors decrease over time? } \\
\text { How does the learning curve look for PA / ACC? } \\
\text { How long does it take to learn what PA / ACC can and cannot? } \\
\text { How often do drivers search for PA / ACC in the digital user manual? } \\
\text { What is the using conditions for PA / ACC? } \\
\text { Does PA / ACC uses efficiently (suitable speed, under recommended } \\
\quad \text { conditions)? } \\
\text { Does user able to turn PA on/off quickly? } \\
\text { What errors do users make? How often do they make errors? } \\
\text { Are there many users who developed a habit of using PA / ACC } \\
\text { most of the time? } \\
\text { How many users do use PA / ACC? } \\
\text { Are there any users who tested PA once and never again? } \\
\text { How many users developed the habit of deactivating PA / ACC? } \\
\text { What time does the system need to respond to the user's request? } \\
\text { How often does system unavailable, due to external factors (road } \\
\text { conditions, bad visibility, etc.)? } \\
\text { How often were PA and ACC deactivated per one profile (in one journey, } \\
\text { in a period of time)? } \\
\text { Who does initiate deactivations (user's decision or the system's disability } \\
\text { to provide service)? }\end{array}$ & $\begin{array}{l}\text { Vehicle identification signals: } \\
\text { Vehicle_ID } \\
\text { Model } \\
\text { Market } \\
\text { Fleet_type } \\
\text { Drive cycle identification signals: } \\
\text { File_ID } \\
\text { Date } \\
\text { Time_At_Start } \\
\text { Total_Drv_Time (min) } \\
\text { Total_Drv_Distance (km) } \\
\text { User identification signals: } \\
\text { Driver_Profile } \\
\text { Key_Used } \\
\text { Seat_Memory_Activated } \\
\text { Seat_Manual_Position } \\
\text { Function status signals (ACC,PA): } \\
\text { Function_Act/Deact_Status (0/1) } \\
\text { Function_Act/Deact_Time (min) } \\
\text { Speed_On_Start(kmh) } \\
\text { Activation_Duration_Distance (km) } \\
\text { Activation_In_Drive_Cycle (\%) } \\
\text { Other signals: } \\
\text { Number_Of_Er_messages } \\
\text { Type_Of_Er_messages } \\
\text { User_Manual_on } \\
\text { GPS_Location }\end{array}$ \\
\hline
\end{tabular}

Figure 4. Example of the Error rate attribute's support by the WICE-data through the usability evaluation questions

According to the case study design, in order to measure usability we need to evaluate User Performance, System Performance, and User Perception (see Figure 5). Usability can be calculated as the mean sum of its dimensions:

$$
U=\frac{1}{n} \sum_{i=1}^{n} f\left(d_{i}\right), \%
$$

where $d_{i}$ is the usability dimension; $\mathrm{n}$ is the number of dimensions.

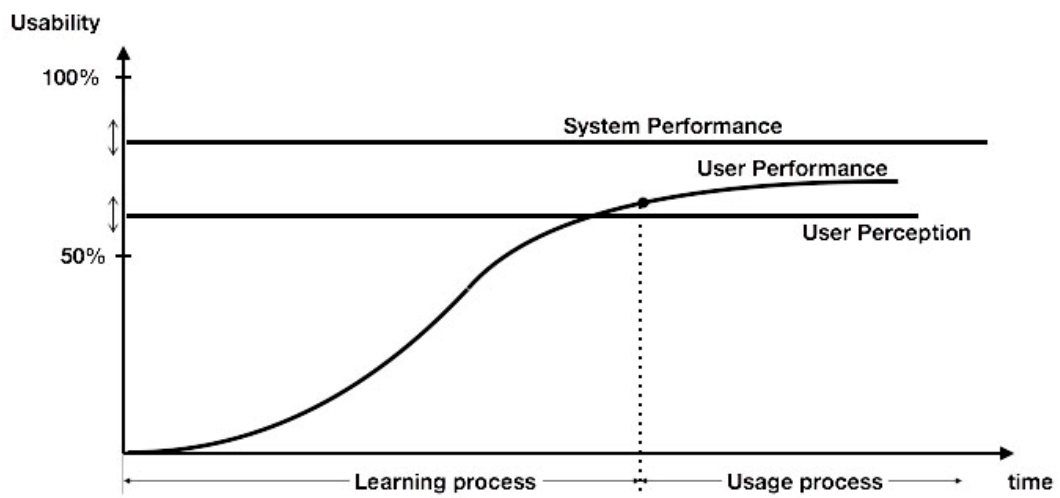

Figure 5. Three usability dimensions: User Performance, System Performance, and User Perception

User Performance, System Performance, and User Perception can in turn also be calculated as mean sums of their dimensions. The same logic could be used for all usability attributes. Moreover, we presume that all usability groups (User Performance, System Performance, and User Perception) have equal impact on usability, and also that all attributes have the same impact inside their groups.

To normalize all attributes, we need to estimate them in percentage. For attributes that depend on time, such as Learnability, Usefulness, System reliability or System robustness - it is easy to determine their values, since we can compare results to the whole time of the drive cycle. However, for attributes such as Error rate or Effectiveness, we need to compare results to values of the worst user performance case 
(for Error rate evaluation) or to values of the professional's performance (in case of Effectiveness evaluation). We found that this data was available from previous LAB-evaluations, although we do not exclude the possibility that additional data input may be required.

\subsection{User performance}

User performance is a group of attributes that measure the specific user behaviour while interacting with the UI (User Interface). As previously mentioned, User Performance could be described by the two following attributes: Learnability and Ease of use.

\subsubsection{Learnability}

In this research we see Learnability as the multidimensional attribute, since we can measure improvement of almost any process over time (Albert and Tullis, 2013). Figure 6 shows possible dimensions that could be set for Learnability evaluation:

- increase/decrease of usage of function over time;

- increase/decrease of mistakes made by the user while using the function over time;

- increase/decrease of searching for help (digital manual in the car).
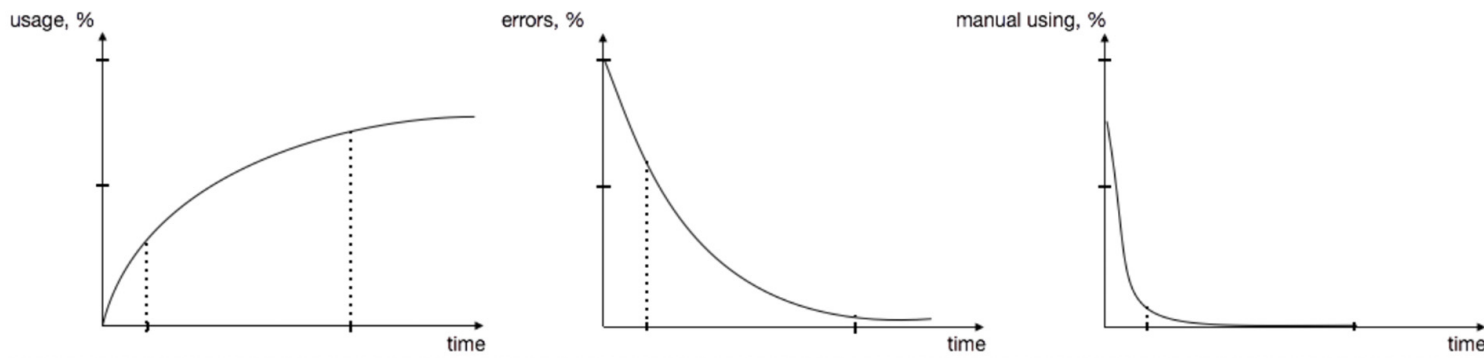

Figure 6. Example of possible dimensions for learnability assessment

With the assessment of Learnability it is possible to answer questions such as how much time does the average user need to learn the system's functionality and how do the user's skills develop over time? The obtained values can help us to conclude how difficult the system was for the average user to learn. When examining the Learnability attribute it is important to measure the improvement of skills of the same driver. Potentially if anyone else would use the car while the measurement process is in progress, the Learnability-curve would be biased by the skills of the other person. Therefore, we decided to identify the user through the sum of first level parameters for user identification, such as driver profile, the key used, seat memory activated, and manual seat position.

\subsubsection{Ease of use}

The "Ease of use" attribute represents the UI usage process and, as can be seen in Figure 2, evaluates by following sub-attributes: Effectiveness, Efficiency, Error rate and Usefulness (see Table 1). We evaluate Effectiveness by looking at the number of parameters that represent customer behaviour regarding particular function and evaluate whether the driver uses a system effectively (at a suitable speed, under recommended conditions, etc.). Error rate attribute measurement contributes to the knowledge of how difficult the Driver Support system is for a user. We evaluate what errors users make, and how often. Usefulness evaluates the necessity of the function itself while looking at the degree (time and frequency of functions activation) to which a function is used by specific users. This information allows us to understand how useful the Driver Assist system appears to drivers and how trustful they are of its quality and performance.

However, the "blind" evaluation (based only on data without physical supervision) makes evaluation of the time that the user required to complete the task impossible. Technically we can measure the time spent on task completion, but we are not aware if the user was concentrating only on performing the particular task. In many cases, multiple tasks demand user involvement at the same time. Moreover, external parameters could also distract the user (road situation that requires immediate action, phone 
call, etc.). Therefore, it is important to exclude "guess" factors, by excluding misleading parameters from the evaluation.

In this study we also met limitations regarding measuring Efficiency. We cannot register all clicks within the graphical user interface, only those that lead to a changing of hardware status. As a result, we are not currently able to measure the number of steps needed to complete the task, and therefore we cannot see if the users learned the optimal path to activate the function. Consequently, the Efficiency attribute should be assessed together with the User Perception attributes with the help of traditional inquiry UEMs, such as surveys, questionnaires or interviews.

\subsection{System performance}

The System Performance attributes are a subset of attributes that evaluate the quality of the Driver Assist system. These are based on system reliability, system responsiveness, and system robustness. In our case, to be able to evaluate the System Performance we need to divide the System into the software system that interacts with the driver and enables the hardware system, and the hardware system that provides driving assistance. If the software system evaluates, regardless of the user, behaviour or external conditions, the hardware system depends on conditions that the driver chooses for the function's activation. Thus, in this study the performance of the software system is evaluated with the help of System robustness and System Responsiveness attributes, and the performance of the hardware system is evaluated with the help of System Reliability attribute.

To illustrate the usage of big data for Usability assessment, data-based analysis for System Reliability attribute evaluation is shown in Figures 7 and 8. Figure 7 represents the monthly mean values for ACC/PA during the period June-December 2017.

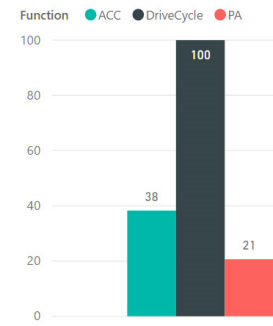

$2017-06$

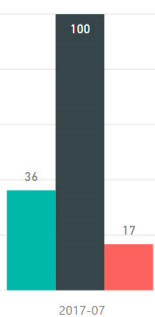

$2017-07$

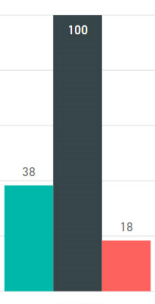

2017-08
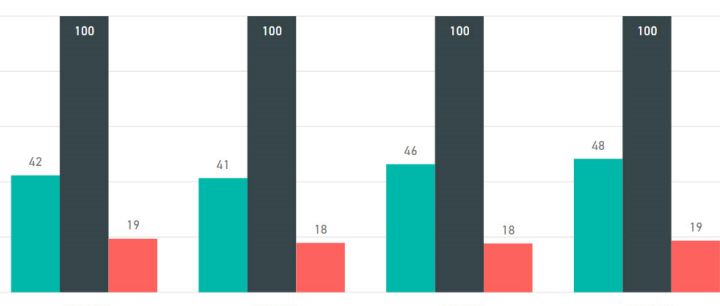

2017-11
$2017-12$

Figure 7. Average of ACC/PA activation time for June-December 2017

However, if we investigate more deeply the results for PA function, trying to separate the user request for $P A$ activation and the $P A$ activation (when physical status changed), we see some interesting findings: users were sending requests for PA activation much more often than the system was able to provide the function (see Figure 8).
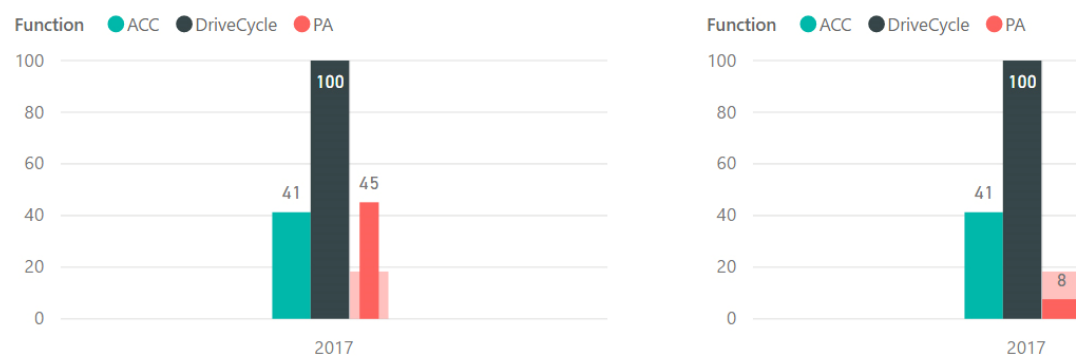

Figure 8. Comparison of the average of users' requests for $P A$ activation (left) and $P A$ activations (right) for June-December 2017

Taking users' requests for PA as $100 \%$ (all potentially possible activations), we got PA function activation only in $17 \%$ of cases. Therefore, the System Reliability for PA function in our case is $17 \%$. Two different reasons for relatively low System Reliability need to be further investigated: user-oriented (users are not activating the function under correct usage conditions (exp., too low speed for activation)) 
and the technology-oriented (technical equipment is unavailable to provide the function (exp., ACC or lane detection, which are prerequisites for PA functioning, are not available)). Therefore including some additional signals, such as ACC, lane detection, and speed at the moment of requests for PA can better explain results for System Reliability of PA function.

It is important to mention that some attributes of User performance, such as Effectiveness or Usefulness depend on the ability of the Driver Support system to provide its functions. Therefore, User performance should be examined together with System performance, in order to investigate how bad/good system performance affects the user behaviour. For example, we can cluster users who decided not to use the function after the few attempts they made. If data shows that the Driver Assist system's performance during those attempts was bad, it can explain why users were dissatisfied with the function. However, if data shows that system performance during those attempts was good and the user still decided negatively, then this cluster of users is interesting to detect for further investigation.

\subsection{User perception}

The goal with User Perception attributes is to gather subjective impressions, preferences or opinions about various aspects of the evaluated system or interface. This knowledge is useful for future improvements to the interface (Ivory and Hearst, 2001). As we mentioned before, in this particular study we cannot measure feelings, user's emotions or cognitive abilities of different users by using any specific equipment, due to the nature of the study (data-based field evaluation without supervision). Therefore, attributes such as Holistic appearance, Consistency, Readability, Perceived driver safety, Understandability, Accessibility, and Satisfaction (see Table 1) need to be assessed by traditional inquiry methods, such as surveys, questionnaires or interviews, to collect supplementary information, regarding User Perception.

According to our case study design, we plan to evaluate User Perception after the customer has been introduced to the system and has used it for some time. Therefore, we are planning to receive customer feedback related to the usage process. In order to include User Perception as a third dimension in Usability Evaluation (see Figure 5), it needs to be transferred to the compatible values (\%) and calculated according to (1) as the mean sum of its dimensions.

\section{Conclusion}

Despite the fact that not all usability attributes can be evaluated with WICE-data there is a significant advantage in the implementation of a data approach for usability attributes assessment. One of the benefits of involving data in subjective evaluation procedures is the fact that artificial intelligence does not tend to "forget" or "generalize" any issues, while the human ability to recall the required information after the interaction has finished is significantly low (Schaeffer et al., 2014).

However, users often have difficulties in estimating the time they spend using a particular application or the time the system needs to respond to their command. Moreover, users tend to estimate their own actions and choices inappropriately. In this case, big data analysis allows more accurate feedback in comparison to user estimation.

In this paper, we investigated the applicability of big data analysis for usability attributes assessment. We presented an approach that is based on the integration of real user data analysis into the subjective evaluation of HMI. We discovered that the data can feed the existing usability structures and the method can increase the usability issues detection, allowing measurement of their magnitude by clustering users with similar behaviour. We present the case study design for Driver Support system assessment, validated by the industry professionals. The next steps will be implementation of this design and reporting the obtained results.

\section{References}

Alpaydin, E. (2010), Introduction to Machine Learning, 2nd ed., The MIT Press, London England.

Becerril, L., Stahlmann, J.T., Beck, J. and Lindemann, U. (2017), "Usability of processes in engineering design", Proceedings of the 21st International Conference on Engineering Design (ICED 17), Vancouver, Canada, 2125.08. 2017., The Design Society, Glasgow.

Berman, J.J., (2013), Principles of big data: preparing, sharing, and analyzing complex information, Newnes. 
Brown, B., Bughin, J., Byers, A.H., Chui, M., Dobbs, R. et al. (2011), Big data: the next frontier for innovation, competition, and productivity, McKinsey Global Institute.

Bruno, V. and Al-Qaimari, G. (2004), "Usability attributes: An initial step toward effective user-centred development", The Proceedings of Australian Computer Human Interaction Conference.

Chalmers, M. (2004), "A historical view of context", Computer Supported Cooperative Work, Vol. 13 No. 3, pp. 223-247. https://doi.org/10.1007/s10606-004-2802-8

Chen, M., Mao, S. and Liu, Y. (2014), "Big data: A survey", Mobile Networks and Applications, Vol. 19 No. 2 , pp. 171-209. https://doi.org/10.1007/s11036-013-0489-0

Cox, M. and Ellsworth, D. (1997), "Managing big data for scientific visualization”, ACM Siggraph, Vol. 97, pp. 21-38.

Diaby, M., Cruz, J.M. and Nsakanda, A.L. (2013), "Shortening cycle times in multi-product, capacitated production environments through quality level improvements and setup reduction”, European Journal of Operational Research, Vol. 228 No. 3, pp. 526-535. 10.1016/j.ejor.2013.02.026

Dumas, J.S. and Redish, J. (1999), A practical guide to usability testing, Intellect books.

Farris, P.W., Bendle, N., Pfeifer, P. and Reibstein, D. (2010), Marketing metrics: The definitive guide to measuring marketing performance, Pearson Education.

Folmer, E. and Bosch, J. (2004), "Architecting for usability: a survey", Journal of systems and software, Vol. 70 No. 1, pp. 61-78. 10.1016/s0164-1212(02)00159-0

Gantz, J. and Reinsel, D. (2011), Extracting value from chaos, IDC iview.

Hashem, I.A.T., Yaqoob, I., Anuar, N.B., Mokhtar, S., Gani, A. and Khan, S.U. (2015), "The rise of "big data" on cloud computing: Review and open research issues", Information Systems, Vol. 47, pp. 98-115. https://doi.org/10.1016/j.is.2014.07.006

Henry, S.L., Abou-Zahra, S. and Brewer, J. (2014), "The role of accessibility in a universal web", Proceedings of the 11th Web for All Conference, ACM, NY, USA. https://doi.org/10.1145/2596695.2596719

IEEE Standards Coordinating Committee (1990), IEEE Standard Glossary of Software Engineering Terminology, IEEE. https://doi.org/10.1109/ieeestd.1990.101064

Ivory, M.Y. and Hearst, M.A. (2001), "The state of the art in automating usability evaluation of user interfaces", ACM Computing Surveys, Vol. 33 No. 4, pp. 470-516. https://doi.org/10.1145/503112.503114

Johanson M. (2017), WICE: Automotive telematics, fleet management, rapid prototyping and software download for test vehicles. [online] Alkit Communications AB. Available at: http://www.alkit.se/doc/WICEwhitepaper.pdf (accessed 05.10.2017).

Klare, G.R. (2000), “The measurement of readability”, ACM Journal of Computer Documentation, Vol. 24 No. 3 , pp. 107-121. https://doi.org/10.1145/344599.344630

Law, E.L.C., Roto, V., Hassenzahl, M., Vermeeren, A.P. and Kort, J. (2009), "Understanding, scoping and defining user experience: a survey approach", Proceedings of the SIGCHI conference on human factors in computing systems, ACM, pp. 719-728. https://doi.org/10.1145/1518701.1518813

Molich, R., Thomsen, A.D., Karyukina, B., Schmidt, L., Ede, M. et al. (1999), "Comparative evaluation of usability tests", CHI'99 extended abstracts on Human factors in computing systems, ACM, pp. 83-84. https://doi.org/10.1145/632716.632768

Nielsen, J. (1994), Usability engineering, Elsevier.

Ovaska, S. (1991), "Usability as a Goal for the Design of Computer Systems", Scandinavian Journal of Information Systems, Vol. 3 No. 1.

Paige, R.F., Kokaly, S., Cheng, B., Bordeleau, F., Storrle, H. et al. (2017), "User experience for model-driven engineering: Challenges and future directions", ACM/IEEE 20th International Conference on Model Driven Engineering Languages and Systems, Institute of Electrical and Electronics Engineers. https://doi.org/10.1109/models.2017.5

Peham, M., Breitfuss, G. and Michalczuk, R. (2014), "The ecoGator app: gamification for enhanced energy efficiency in Europe", Proceedings of the Second International Conference on Technological Ecosystems for Enhancing Multiculturality, ACM, pp. 179-183. https://doi.org/10.1145/2669711.2669897

Schaeffer, J. and Carlsson, A.L. (2014), "The method of photo-elicitation from a phenomenological perspective", Proceedings of the DESIGN 2014 / 13th International Design Conference, pp. 49-58.

Tullis, T. and Albert, B. (2013), "Performance Metrics", In: Tullis, T. and Albert, B., Measuring the User Experience: Collecting, Analyzing, and Presenting Usability Metrics, 2nd ed., Morgan Kaufmann, Boston, pp. 63-97. https://doi.org/10.1016/b978-0-12-415781-1.00004-2

Julia Orlovska, Ph.D. candidate

Chalmers University of Technology, Industrial and Materials Science

Fullblodsgatan 5a, lgh 1201, 43132 Mölndal, Sweden

Email: orlovska@chalmers.se 